\title{
INVARIANT RADON TRANSFORMS ON A SYMMETRIC SPACE
}

\author{
JEREMY ORLOFF
}

\begin{abstract}
Injectivity and support theorems are proved for a class of Radon transforms, $R_{\mu}$, for $\mu$ a smooth family of measures defined on a certain space of affine planes in $\mathbb{X}_{0}$, where $\mathbb{X}_{0}$ is the tangent space, of a Riemannian symmetric space of rank one. The transforms are defined by integrating against $\mu$ over these planes. We show that if $R_{\mu} f$ is supported inside a ball of radius $R$ then so is $f$. This is true for $f \in L_{c}^{2}\left(\mathbb{X}_{0}\right)$ or $f \in \mathscr{E}^{\prime}\left(\mathbb{X}_{0}\right)$. Furthermore, $R_{\mu}$ is invertible on either of these domains. The main technique is to use facts about spherical harmonics to reduce the problem to a one-dimensional integral equation.
\end{abstract}

\section{INTRODUCTION}

Radon transforms have been extensively studied. See, for example, Guillemin [2], Helgason [6], and Gelfand [1]. In this paper we will generalize and unify several results on Radon transforms. If a compact group $K$ acts on a real vector space $\mathbb{X}_{0}$, then one has the following notion of a $K$-invariant Radon transform. The ingredients are $\Xi_{0}$, the space of affine hyperplanes, $F=\{(x, \xi) \mid x \in \xi\} \subset \mathbb{X}_{0} \times \Xi_{0}$, and a smooth function $\mu$ on $F$. The Radon transform is defined for $f \in L_{c}^{2}\left(\mathbb{X}_{0}\right)$ by $R_{\mu} f(\xi)=\int_{x \in \xi} f(x) \mu(x, \xi) d x .(d x$ is Lebesgue measure.) If $R_{\mu}$ intertwines the actions of $K$ it is called $K$ invariant. This is easily seen to be equivalent to the condition $\mu(k \cdot x, k \cdot \xi)=\mu(x, \xi)$ for all $k \in K$ and $x \in \xi$.

It is natural to ask if $R_{\mu}$ is invertible and to look for support theorems. Quinto [11] covers the case when $K=O(n)$ and $\mathbb{X}_{0}=\mathbb{R}^{n}$. He proves a support theorem and invertibility for $K$-invariant Radon transforms. Also, Helgason [3] proves support and injectivity theorems in the case $\mu \equiv 1$. Helgason [5] proves a support theorem for the classical Radon transform on Euclidean space.

If $\mathbb{X}_{0}$ has a natural complex or quaternionic structure one can play the same game with $\Xi_{\mathbb{F}}$, the space of complex or quaternionic hyperplanes. ( $\mathbb{F}$ is either the complex numbers $\mathbb{C}$ or the quaternions $\mathbb{H}$.) Quinto [10] covers the case when $K=U(n)$ and $\mathbb{X}_{0}=\mathbb{C}^{n}$. Once again he proves injectivity and a support theorem.

In this paper we will take $\mathbb{X}_{0}$ to be the tangent space of a rank one Rie-

Received by the editors July 1, 1988.

1980 Mathematics Subject Classification (1985 Revision). Primary 44A15; Secondary 43A85. 
mannian symmetric space and $K$ to be the isotropy subgroup. Our main results, Theorem 3.2 and Corollary 6.5, provide a support theorem and prove invertibility for $K$-invariant Radon transforms. We also obtain support and invertibility results for $R_{\mu}$ acting on distributions (Theorem 7.1 and Corollary 7.4).

We have used the group theory to present a unified picture of the transforms described above. Our technique of using the dual transform to reduce the proof of the support theorem to solving an Abel integral equation originates with Quinto [11]. Also, Helgason [3, pp. 105-112] uses an Abel integral equation to prove support theorems in the case $\mu \equiv 1$. One interesting feature of this is that the complex and quaternionic results follow from the real case. This is true because the spaces of real and complex affine hyperplanes are essentially isomorphic. (See Lemma 6.2.) The group theoretic approach also allows us to define Radon transforms on the symmetric space itself. There is also a natural generalization to higher rank spaces. Finally, one can investigate different representations of $K$. We will return to these questions in another paper.

The outline of the paper is as follows. In $\S 1$ we collect notation from the theory of symmetric spaces and we relate it to the familiar language of points and planes. Finally, we define the Radon transform and its dual. In $\S 2$ we justify the use of the term dual transform. In $\S 3$ we state our main theorem in the real case. $\S 4$ is devoted to reviewing known facts about harmonic polynomials, especially their decomposition under the action of $K$. In $\S 5$ we prove the main theorem. Using the results in $\S \S 2$ and 4 the proof reduces to a one-dimensional integral equation of Volterra or Abel. In $\S 6$ we change fields. After relating the real and $\mathbb{F}$ hyperplane spaces and the real and $\mathbb{F}$ dual Radon transforms we extend the results of $\S 3$. Finally, in $\S 7$ we extend the results of $\S \S 3$ and 6 to distributions.

\section{Notation}

We collect here the notation we will use throughout this paper. In the "flat case" we have the option of using either group theoretic notation or the more familiar terminology of points and planes. We list both notations, along with a brief dictionary relating the two. Also, note for the flat case the machinery of semisimple Lie groups is not really used (although it will play a role in later sections.)

We start with the standard notation for symmetric spaces. (See Helgason [4].) Let $G$ be a connected semisimple Lie group with finite center and Lie algebra $\mathfrak{g}$. Fix $K$, a maximal compact subgroup of $G$ and let $f$ denote its Lie algebra. We denote the Killing form on $\mathfrak{g}$ by $\langle$,$\rangle . Let \mathfrak{p}$ be the orthogonal complement to $\mathfrak{f}$ with respect to $\langle$,$\rangle . Thus \mathfrak{g}=\mathfrak{f}+\mathfrak{p}$. Under the adjoint action, $K$ preserves $\mathfrak{p}$. We denote this action by a dot, that is, $\operatorname{Ad}(k) X=k \cdot X$ for $k \in K$ and $X \in \mathfrak{p}$. In general, we will use a dot to describe a group action whenever the intended action is clear from the context.

The Cartan motion group $K \ltimes \mathfrak{p}$ is denoted by $G_{0}$. Let $\mathbb{X}=G / K$ and 
$\mathbb{X}_{0}=G_{0} / K$. Then $\mathbb{X}$ and $\mathbb{X}_{0}$ are symmetric spaces. $\mathbb{X}$ is a Riemannian symmetric space of noncompact type and $\mathbb{X}_{0} \approx \mathfrak{p}$ is its flat analogue. (We consider $\mathbb{X}_{0}$ to be the tangent space of $\mathbb{X}$ at the origin $o=e K \in G / K$.)

Fix a a maximal abelian subalgebra of $\mathfrak{p}$. Let $M=Z_{K}(\mathfrak{a})$ be the centralizer of $\mathfrak{a}$ in $K$, and let $M^{\prime}=N_{K}(\mathfrak{a})$ be the normalizer of $\mathfrak{a}$ in $K$. Let $\mathfrak{q}$ be the orthogonal complement of $\mathfrak{a}$ inside $\mathfrak{p}$ with respect to $\langle$,$\rangle . Let P_{\mathfrak{a}}$ be the orthogonal projection to $\mathfrak{a}$.

The dual spaces of $\mathbb{X}$ and $\mathbb{X}_{0}$ are, respectively, $\Xi$, the space of horocycles in $\mathbb{X}$, and $\Xi_{0}$, the space of "flat horocycles" consisting of all $G_{0}$ translates of $\mathfrak{q}$. Thus, $\Xi_{0}$ is a set of affine planes in $\mathfrak{p}$. (If $\operatorname{dim} \mathfrak{a}=1$ then $\Xi_{0}$ is the space of all affine hyperplanes.) As a homogeneous space $\Xi_{0}=G_{0} /\left(M^{\prime} \ltimes \mathfrak{q}\right)$. To describe $\Xi$ we need to choose an Iwasawa decomposition $G=K A N$ (see Helgason [4]). As a homogeneous space $\Xi=G / M N$.

In summary, we have

$$
\begin{aligned}
& \mathfrak{g}=\mathfrak{f}+\mathfrak{p}, \quad G_{0}=K \ltimes \mathfrak{p}, \quad \mathbb{X}=G / K, \quad \mathbb{X}_{0}=G_{0} / K \approx \mathfrak{p}, \\
& \mathfrak{p}=\mathfrak{a}+\mathfrak{q}, \quad M=Z_{K}(\mathfrak{a}), \quad \Xi=G / M N, \quad \Xi_{0}=G_{0} /\left(M^{\prime} \ltimes \mathfrak{q}\right), \\
& P_{\mathfrak{a}}=\text { orthog. proj. to } \mathfrak{a}, \quad M^{\prime}=N_{K}(\mathfrak{a}) \text {. }
\end{aligned}
$$

For $\xi \in \Xi_{0}$ we let $\hat{\xi}=\{\mathbb{X} \in \mathfrak{p} \mid X \in \xi\}$. For $X \in \mathfrak{p}$ we let $\check{X}=\left\{\xi \in \Xi_{0} \mid X \in\right.$ $\xi$. So, $\hat{\xi}=$ the points of $\xi$ and $\check{X}=$ the set of flat horocycles through $X$. We make similar definitions for the curved spaces $\mathbb{X}$ and $\Xi$.

Consider the set $F_{0}=\left\{(X, \xi) \in \mathbb{X}_{0} \times \Xi_{0} \mid X \in \xi\right\}$. It is easy to see that $F_{0}$ is isomorphic to $G_{0} / M^{\prime}$ via the map

$$
g M^{\prime} \rightarrow(g \cdot 0, g \cdot \mathfrak{q}) .
$$

Similarly, in the curved case we get $F=G / M$. Thus we have the double fibrations

$$
\begin{array}{cccc}
G_{0} / M^{\prime}=F_{0} & \multicolumn{2}{c}{G / M=F} \\
p_{1} \swarrow & p_{2} & p_{1} \swarrow & \searrow p_{2} \\
X & \Xi & X & \Xi .
\end{array}
$$

Of course, $\check{X}=p_{2} p_{1}^{-1}(X)$ and $\hat{\xi}=p_{1} p_{2}^{-1}(\xi)$.

The set of flat horocycles through the origin is just

$$
\check{0}=\{k \cdot \mathfrak{q} \mid k \in K\} \approx K / M^{\prime} \text {. }
$$

In general,

$$
\check{X}=\{X+k \cdot \mathfrak{q} \mid k \in K\} \approx K / M^{\prime} .
$$

Thus, we have a canonical measure $d_{x} \xi$ on $\check{X}$ coming from the $K$ invariant measure on $K / M^{\prime}$ (of total volume one). Likewise, the flat horocycle $\xi=$ $Z+k \cdot \mathfrak{q}$ has

$$
\hat{\xi}=\{Z+k \cdot Y \mid Y \in \mathfrak{q}\} \approx \mathfrak{q} .
$$

Hence, it carries a canonical measure $d_{\xi} X$ coming from Lebesgue measure on q. 
Let $\mu$ be a smooth function on $F_{0}$. Take $f \in C_{c}\left(\mathbb{X}_{0}\right)$ and $\varphi \in C\left(\Xi_{0}\right)$. We define the Radon transform and its dual by

$$
\begin{aligned}
R_{\mu} f(\xi) & =\int_{X \in \hat{\xi}} f(X) \mu(X, \xi) d_{\xi} X, \\
R_{\mu}^{*} \varphi(X) & =\int_{\xi \in \dot{X}} \varphi(\xi) \mu(X, \xi) d_{X} \xi .
\end{aligned}
$$

Let $\xi=Z+k \cdot \mathfrak{q}$. Using the descriptions of $\hat{\xi}, \check{X}$, and their measures given above we get:

$$
\begin{aligned}
R_{\mu} f(\xi) & =\int_{Y \in \mathfrak{q}} f(Z+k \cdot Y) \mu(Z+k \cdot Y, Z+k \cdot \mathfrak{q}) d Y, \\
R_{\mu}^{*} \varphi(X) & =\int_{K} \varphi(X+k \cdot \mathfrak{q}) \mu(X, X+k \cdot \mathfrak{q}) d k .
\end{aligned}
$$

Here $d k$ is the Haar measure on $K$ of total volume one.

The Killing form provides a metric on $\mathfrak{p}$. For a plane in $\Xi_{0}$ its norm is defined as its distance from the origin. Thus $|k \cdot(H+q)|=|H|$. We will let $B^{R}$ denote the ball of radius $R$ inside either $\mathfrak{p}$ or $\Xi_{0}$.

We now rewrite the Radon transforms using strictly group theoretic notation. For $Y \in \mathfrak{p}$ we let $T_{Y}$ be the corresponding translation in $G_{0}$. Let $\xi_{0}=$ $e\left(M^{\prime} \ltimes \mathfrak{q}\right) \in \Xi_{0}$. ( $\xi_{0}$ is the horocycle $\left.\mathfrak{q}.\right)$ Fix a smooth function, $\mu$, on $F_{0}=G_{0} / M^{\prime}$. For $f$ and $\varphi$ as before and $g \in G_{0}$ we have

$$
R_{\mu} f\left(g \cdot \xi_{0}\right)=\int_{\mathfrak{q}} f\left(g \cdot T_{Y} \cdot 0\right) \mu\left(g \cdot T_{Y} M^{\prime}\right) d Y,
$$

and

$$
R_{\mu}^{*} \varphi(g \cdot 0)=\int_{K} \varphi\left(g \cdot k \cdot \xi_{0}\right) \mu\left(g \cdot k M^{\prime}\right) d k .
$$

This notation carries over easily to the curved case. Take $\mu \in C(G / M)$, $f \in C_{c}(\mathbb{X})$, and $\varphi \in C(\Xi)$. Then

$$
\begin{aligned}
R_{\mu} f(g M N) & =\int_{N} f(g n K) \mu(g n M) d n, \\
R_{\mu}^{*} \varphi(g K) & =\int_{K} \varphi(g k M N) \mu(g k M) d k .
\end{aligned}
$$

From now on we will restrict our attention to the flat case. The curved case is more subtle. We hope to return to it in another paper.

Example. Let $G=\mathrm{SO}_{e}(n, 1)$. Then $K=\mathrm{SO}(n), \mathbb{X}_{0}=\mathfrak{p} \approx \mathbb{R}^{n}, \mathfrak{a}$ is any line through the origin, $\Xi_{0}$ is the space of all affine hyperplanes, and the action of $K$ on $\mathfrak{p}$ is the usual one. For $\mu=1, R_{\mu}$ is the classical Radon transform. If $\mu$ is $K$ invariant (see $\S 3$ ) we are in the case of Quinto [11].

\section{Duality}

We have referred to $R_{\mu}$ and $R_{\mu}^{*}$ as dual transforms. In this section we will make this precise. 
First we need "coordinates" and measures on $\mathbb{X}_{0}$ and $\Xi_{0}$. For $\mathbb{X}_{0}=\mathfrak{p}$, we have Lebesgue measure $d X$. Clearly $d X$ is $G_{0}$ invariant. We also have polar coordinates (see Helgason [4])

$$
\Phi: K / M \times \mathfrak{a} \rightarrow \mathfrak{p} \text { by } \Phi(k M, H)=k \cdot H .
$$

The map $\Phi$ is surjective and generically $w$ to one $\left(w=\#\left(M^{\prime} / M\right)\right)$.

For $\Xi_{0}$, we have polar coordinates

$$
\Psi: K / M \times \mathfrak{a} \rightarrow \Xi_{0} \text { by } \Psi(k M, H)=k \cdot(H+\mathfrak{q}) .
$$

The map $\Psi$ is surjective and generically $w$ to one. Using these polar coordinates, we get a $G_{0}$-invariant measure on $\Xi_{0}$ given by

$$
\int_{\Xi_{0}} \varphi(\xi) d \xi=\int_{K \times \mathfrak{a}} \varphi(k \cdot(H+\mathfrak{q})) d k d H .
$$

The following lemma is proved in Helgason [7] in the case $\mu \equiv 1$.

Lemma 2.1. The Radon transforms $R_{\mu}$ and $R_{\mu}^{*}$ are dual. That is, for $f \in$ $L_{c}^{2}\left(\mathbb{X}_{0}\right)$ and $\varphi \in L^{2}\left(\Xi_{0}\right)$. (The subscript $c$ indicates compact support.) We have

$$
\int_{\Xi_{0}} R_{\mu} f(\xi) \cdot \varphi(\xi) d \xi=\int_{\mathrm{X}_{0}} f(X) \cdot R_{\mu}^{*} \varphi(X) d X .
$$

Proof. The left-hand side of equation (2.1) equals

$$
\int_{K \times \mathfrak{a}} \int_{\mathfrak{q}} f(k(H+Y)) \cdot \varphi(k(H+\mathfrak{q})) \mu(k(H+Y), k(H+\mathfrak{q})) d Y d k d H .
$$

The right-hand side of (2.1) equals

$$
\begin{aligned}
\int_{X_{0}} \int_{K} f(X) \cdot \varphi(X+k \cdot \mathfrak{q}) \mu(X, X+k \cdot \mathfrak{q}) d k d X \\
=\int_{K} \int_{\mathbb{X}_{0}} f(k \cdot X) \cdot \varphi(k \cdot X+k \cdot \mathfrak{q}) \mu(k \cdot X, k \cdot X+k \cdot \mathfrak{q}) d X d k \\
=\int_{K} \int_{\mathfrak{q}} \int_{\mathfrak{a}} f(k(H+Y) \varphi(k(H+Y+\mathfrak{q})) \\
\times \mu(k(H+Y), k(H+Y+\mathfrak{q})) d Y d H d k .
\end{aligned}
$$

The first equality follows from Fubini's theorem and the $K$ invariance of the measure $d X$. Since $Y+\mathfrak{q}=\mathfrak{q}$ for $Y \in \mathfrak{q}$, the last formula equals the formula for the left-hand side given above.

\section{MAIN THEOREM}

In this section we will state our main theorem. First we must define the notion of $K$ invariance.

$K$ invariance: Let $L_{k}$ denote translation by $k \in K$ on $\mathbb{X}_{0}$ or on $\Xi_{0}$. 
Lemma 3.1. The following are equivalent for all $k \in K$.

(1) $\mu(k \cdot X, k \cdot(X+\mathfrak{q}))=\mu(X, X+\mathfrak{q})$.

(2) Considering $\mu$ as a function on $G_{0} / M^{\prime}, \mu\left(k \cdot g M^{\prime}\right)=\mu\left(g M^{\prime}\right)$.

(3) $R_{\mu}\left(f \circ L_{k}\right)=\left(R_{\mu} f\right) \circ L_{k}$ for $f \in C_{c}(\mathfrak{p})$.

(4) $R_{\mu}^{*}\left(\varphi \circ L_{k}\right)=\left(R_{\mu}^{*} \varphi\right) \circ L_{k}$ for $\varphi \in C\left(\Xi_{0}\right)$.

Proof. (1) $\Leftrightarrow(2)$ : This is obvious from equation (1.1).

$(1) \Leftrightarrow(3)$ : We have

$$
R_{\mu}\left(f \circ L_{k^{\prime}}\right)(X+k \cdot \mathfrak{q})=\int_{\mathfrak{q}} f\left(k^{\prime} \cdot(X+k \cdot Y)\right) \mu(X+k \cdot Y, X+k \cdot \mathfrak{q}) d Y .
$$

On the other hand,

$\left(R_{\mu} f\right) \circ L_{k^{\prime}}(X+k \cdot \mathfrak{q})=\int_{\mathfrak{q}} f\left(k^{\prime} \cdot(X+k \cdot Y)\right) \mu\left(k^{\prime} \cdot(X+k \cdot Y), k^{\prime} \cdot(X+k \cdot \mathfrak{q})\right) d Y$.

Showing $(1) \Leftrightarrow(4)$ is similar.

If any of the conditions in Lemma 3.1 hold we say that $\mu, R_{\mu}$, or $R_{\mu}^{*}$ is $K$ invariant.

Assumptions. From now on we will assume $G / K$ has real rank one. This means $\operatorname{dim} \mathfrak{a}=1$. We also make the following assumptions:

(1) $\mu$ is $K$ invariant.

(2) $\mu(H, H+\mathfrak{q}) \neq 0$ for all $H \in \mathfrak{a}$.

(3) $\mu$ is smooth.

(4) For all $H \in \mathfrak{a}$ and $Y \in \mathfrak{q}, \mu(H-Y, H+\mathfrak{q})=\mu(H+Y, H+\mathfrak{q})$.

Remarks. (1) Combined with $K$ invariance assumption (2) says $\mu(X, \xi) \neq 0$ if $X$ is the point on $\xi$ nearest the origin.

(2) Assumption (3) is not strictly necessary. By examining the proof of the main theorem we need only assume $N$ times continuously differentiable for some fixed $N$.

(3) Assuming $K$ invariance, we can state assumption (4) in a number of equivalent ways. For instance, $\mu\left(H+m^{\prime} Y, H+\mathfrak{q}\right)=\mu(H+Y, H+\mathfrak{q})$ for all $H \in \mathfrak{a}, Y \in \mathfrak{q}$, and $m^{\prime} \in M^{\prime}$. Also, $\mu\left(T_{H} m^{\prime} T_{Y} M^{\prime}\right)=\mu\left(T_{H} T_{Y} M^{\prime}\right)$. (See §1.) To show these two equivalences we need facts one and two stated between Lemma 4.1 and Lemma 4.2.

(4) Combined with $K$ invariance assumption (4) is automatic if $m_{2 \alpha}=0$ or $m_{2 \alpha x}>1$. (See $\S 4$.)

We can now state our main theorem. It is a generalization of Helgason's support theorem for the Euclidean Radon transform (see [5]). Our proof is modelled on Quinto [11].

Theorem 3.2 (Support Theorem). Suppose $\mu$ satisfies the assumptions in (3.2) . Then we have

(1) If $f \in L_{c}^{2}\left(\mathbb{X}_{0}\right)$ and $\operatorname{supp} R_{\mu} f \subset B^{R}$ then $\operatorname{supp} f \subset B^{R}$. 
(2) $R_{\mu}: L_{c}^{2}\left(\mathbb{X}_{0}\right) \rightarrow L^{2}\left(\Xi_{0}\right)$ is injective.

Remark. We assume a priori that $f$ is compactly supported. However, this assumption is probably unnecessary. For example, for the classical Radon transform one need only assume a certain growth condition on $f$. (See Helgason [3].)

\section{STRUCTURE THEORY}

The proof of the support theorem requires some more facts and notation about symmetric spaces. In this section we will outline what is needed. For more detail see Helgason [4, 3].

The positive restricted roots of $\mathfrak{a}$ are denoted $\alpha$ and (if it exists) $2 \alpha$. Their multiplicities are denoted $m_{\alpha}$ and $m_{2 \alpha}$ and the corresponding root spaces are denoted $\mathfrak{g}_{\alpha}$ and $\mathfrak{g}_{2 \alpha}$. We let $\theta$ be the Cartan involution corresponding to the decomposition $\mathfrak{g}=\mathfrak{f}+\mathfrak{p}$. (That is, $\theta$ is 1 on $\mathfrak{f}$ and -1 on $\mathfrak{p}$.) We define

$$
\mathfrak{q}_{1}=\left\{X-\theta X \mid X \in \mathfrak{g}_{\alpha}\right\}, \quad \mathfrak{q}_{2}=\left\{X-\theta X \mid X \in \mathfrak{g}_{2 \alpha}\right\} ;
$$

thus,

$$
\mathfrak{q}=\mathfrak{q}_{1}+\mathfrak{q}_{2}=\mathfrak{a}^{\perp} .
$$

As before, $M=Z_{K}(\mathfrak{a})$ and $M^{\prime}=N_{K}(\mathfrak{a})$. Also, we know that $W=M^{\prime} / M$ has cardinality two.

We now fix $0 \neq H_{0} \in \mathfrak{a}$. We know that the orbit $K \cdot H_{0}$ is isomorphic to $K / M$. Let $\mathscr{H}$ denote the harmonic polynomials on $\mathfrak{p}$. Let $\widehat{K}_{M}$ be the set of irreducible representations of $K$ with a unique (up to scalar) $M$ fixed vector. Then we have according to Kostant [8] (see also Helgason [3])

$$
\mathscr{H} \hookrightarrow L^{2}(K / M)
$$

and

$$
\mathscr{H} \approx \bigoplus_{\delta \in \hat{K}_{M}} \mathscr{H}_{\delta},
$$

where the space $\mathscr{H}_{\delta}$ is an irreducible subrepresentation of $\mathscr{H}$ equivalent to $\delta$ and consisting of homogeneous polynomials of degree $d_{\delta}$. The injection of $\mathscr{H}$ into $L^{2}(K / M)$ is accomplished by restricting a polynomial in $\mathscr{H}$ to the orbit $K \cdot H_{0}$. It commutes with the action of $K$ and its image is dense in $L^{2}(K / M)$.

For each $\delta$ there is a unique $M$ invariant polynomial $\varphi_{\delta} \in \mathscr{H}_{\delta}$ such that $\varphi\left(H_{0}\right)=1$. We consider it as either a function of $K / M$ or a harmonic polynomial on $\mathfrak{p}$.

The following "Funk-Hecke theorem" is trivial from the group theoretic viewpoint.

Lemma 4.1. Choose any $Y_{\delta} \in \mathscr{H}_{\delta}$ (considered as a function on $K / M$ by $Y_{\delta}(k)=$ $\left.Y_{\delta}\left(k \cdot H_{0}\right)\right)$; then

$$
\int_{M} Y_{\delta}\left(k_{1} m k_{2}\right) d m=Y_{\delta}\left(k_{1}\right) \varphi_{\delta}\left(k_{2}\right)
$$


Proof. Fix $k_{1} \in K$. Let $g\left(k_{2}\right)=\int_{M} Y_{\delta}\left(k_{1} m k_{2}\right) d m$. Since $\mathscr{H}_{\delta}$ is $K$ stable this function is in $\mathscr{H}_{\delta}$ and it is $M$ invariant. Thus it is a multiple of $\varphi_{\delta}$. To determine which multiple let $k_{2}=e$.

Finally, we need suitable coordinates on the sphere in $\mathfrak{p}, K \cdot H_{0}=K / M$. We will use the $S U(2,1)$ reduction introduced in Helgason [5]. Our calculations involving this reduction contain only minor modifications of calculations in [5]. Choose $0 \neq X_{\alpha} \in \mathfrak{g}_{\alpha}$ and $0 \neq X_{2 \alpha} \in \mathfrak{g}_{2 \alpha}$. (If $m_{2 \alpha}=0$ then there is no $X_{2 \alpha}$.) Define $Z_{1}=X_{\alpha}-\theta X_{\alpha}$ and $Z_{2}=X_{2 \alpha}-\theta X_{2 \alpha}$. We assume $\left|H_{0}\right|=\left|Z_{1}\right|=\left|Z_{2}\right|=$ 1. We know (see Helgason [3]) that the subalgebra generated by $H_{0}, Z_{1}$, and $Z_{2}$ is isomorphic to $\mathfrak{s u}(2,1)$. (If $m_{2 \alpha}=0$ we get $\mathfrak{s l}(2)$.) From Kostant [8] we know the following:

(1) If $m_{2 \alpha}>1$ then $M$ acts transitively on the product of the unit spheres in $\mathfrak{q}_{1}$ and $\mathfrak{q}_{2}$.

(2) If $m_{2 \alpha}=1$ then $M$ acts transitively on the unit sphere in $q_{1}$.

(3) If $m_{2 \alpha} \neq 0$ then $m_{2 \alpha}$ is even and $m_{\alpha}$ is odd.

(4) If $m_{2 \alpha}=0$ then $M$ is transitive on the sphere in $\mathfrak{q}=\mathfrak{q}_{1}$.

Thus we get the following coordinates on $K / M=K \cdot H_{0}$. (Recall that $\left|H_{0}\right|=1$.)

$$
(m, s, b) \rightarrow m\left(s H_{0}+a Z_{1}+b Z_{2}\right) \in K \cdot H_{0} .
$$

Here, if $m_{2 \alpha}>1$ then

$$
-1 \leq s \leq 1, \quad 0 \leq b \leq \sqrt{1-s^{2}}, \quad m \in M, \quad a=\sqrt{1-s^{2}-b^{2}} ;
$$

if $m_{2 x}=1$ then

$$
-1 \leq s \leq 1, \quad-\sqrt{1-s^{2}} \leq b \leq \sqrt{1-s^{2}}, \quad m \in M, \quad a=\sqrt{1-s^{2}}-b^{2} ;
$$

if $m_{2 \ltimes r}=0$ then

$$
-1 \leq s \leq 1, \quad m \in M, \quad a=\sqrt{1-s^{2}}, \quad b=0 .
$$

Now we need to compute the measure on $K / M$ in these coordinates.

Lemma 4.2. For the various cases we get the following formulas for

$$
c \int_{K / M} f(k M) d k \text {. }
$$

$\left(c=A_{\left(m_{n}+m_{2 n}+1\right)} / A_{m_{n}} A_{m_{2 n}}\right.$, where $A_{n}$ is the area of the unit sphere in $\left.\mathbb{R}^{n}.\right)$

$$
\begin{aligned}
& m_{2 \alpha}>1: \\
& \qquad \int_{s=-1}^{1} \int_{b=0}^{\sqrt{1-s^{2}}} \int_{M} f\left(m\left(s H_{0}+a Z_{1}+b Z_{2}\right)\right) a^{\left(m_{n}-2\right)} b^{\left(m_{2 n}-1\right)} d b d s d m,
\end{aligned}
$$




$$
\begin{aligned}
m_{2 \alpha}= & 1: \\
& \int_{s=s-1}^{1} \int_{b=-\sqrt{1-s^{2}}}^{\sqrt{1-s^{2}}} \int_{M} f\left(m\left(s H_{0}+a Z_{1}+b Z_{2}\right)\right) a^{\left(m_{n}-2\right)} d b d s d m, \\
m_{2 \alpha}= & 0: \\
& \int_{s=-1}^{1} \int_{M} f\left(m\left(s H_{0}+a Z_{1}\right)\right) a^{m_{o}-2} d s d m .
\end{aligned}
$$

As before $a=\sqrt{1-s^{2}-b^{2}}\left(\sqrt{1-s^{2}}\right.$ if $\left.m_{2 \alpha}=0\right)$.

Remark. In the case $m_{2 \alpha}=0$ we have assumed $m_{\alpha}>1$ The case $m_{2 \alpha}=0$ and $m_{\alpha}=1$ is easy to handle separately.

Proof. This is a straightforward calculation using the fact that $M$ is transitive on the product of the unit spheres of $\mathfrak{q}_{1}$ and $\mathfrak{q}_{2}$.

We will need the following lemma.

Lemma 4.3. If $k \cdot H_{0}=m\left(s H_{0}+a Z_{1}+b Z_{2}\right)$ then for some $m_{1} \in M k^{-1} \cdot H_{0}=$ $m_{1}\left(s H_{0}-a Z_{1}-b Z_{2}\right)$.

Proof. We reduce the proof to a calculation in $\mathfrak{s u}(2,1)$. Take $k_{1} \in \mathrm{SU}(2,1)$ (the copy of $\mathrm{SU}(2,1)$ associated to $\left(H_{0}, Z_{1}, Z_{2}\right)$ ) such that $k_{1} \cdot H_{0}=s H_{0}+$ $a Z_{1}+b Z_{2}$. This implies $k=m k_{1} m_{2}$ for some $m_{2} \in M$. Thus, $k^{-1}=$ $m_{2}^{-1} k_{1}^{-1} m^{-1}$ and $k^{-1} \cdot H_{0}=m_{2}^{-1} k_{1}^{-1} \cdot H_{0}$. Now an easy calculation inside $\mathrm{SU}(2.1)$ shows $k_{1}^{-1} \cdot H_{0}=m_{3}\left(s H_{0}-a Z_{1}-b Z_{2}\right)$ for some $m_{3} \in M \cap \mathrm{SU}(2,1)$. This proves the lemma.

\section{PROOF OF THE SUPPORT THEOREM}

In this section we will prove the support theorem. First, we note that part (1) implies part (2) by letting $R \rightarrow 0$.

To prove part (1) we consider functions of the following form on $\Xi_{0}$ :

$$
g(k(H+\mathfrak{q}))=Y_{\delta}\left(k \cdot H_{0}\right) g_{\delta}(H)
$$

where

(a) $Y_{\delta} \in \mathscr{H}_{\delta}$ and $g_{\delta}$ is smooth on a,

(b) $\operatorname{supp} g_{\delta} \cap B^{R}=\varnothing$,

(c) $g_{\delta}(-H)=(-1)^{d_{\delta}} g_{\delta}(H)$.

Condition (c) is needed to insure that $g$ is well defined as a function on $\Xi_{0}$.

We will show that every function on $\mathfrak{p}$ of the form

$$
h(k \cdot H)=Y_{\delta}\left(k \cdot H_{0}\right) h_{\delta}(H)
$$

where
(a) $Y_{\delta} \in \mathscr{H}_{\delta}$,
(b) $\operatorname{supp} h_{\delta} \cap B^{R}=\varnothing$, 
(c) $h_{\delta}(-H)=(-1)^{d_{\delta}} h_{\delta}(H)$,

(d) $h_{\delta}$ is smooth and compactly supported on $\mathfrak{a}$, is $R_{\mu}^{*} g$ for some $g$ of the type (5.1) above.

Assuming this, part (1) follows directly: Pick any $h$ of type (5.2) and take $g$ of type (5.1) such that $R_{\mu}^{*} g=h$. Then, since $\operatorname{supp} R_{\mu} f \subset B^{R}$ we have from Lemma 2.1

$$
0=\left\langle R_{\mu} f, g\right\rangle=\left\langle f, R_{\mu}^{*} g\right\rangle=\langle f, h\rangle .
$$

But the span of the functions $h$ of type (5.2) ( $\delta$ is allowed to vary) is dense in $L^{2}\left(\mathbb{X}_{0}-B^{R}\right)$. Thus $f$ is perpendicular to $L^{2}\left(\mathbb{X}_{0}-B^{R}\right)$. This implies supp $f \subset B^{R}$, proving part (1).

We now have to show that $R_{\mid}^{*} \mu$ maps functions of type (5.1) onto those of type (5.2). (Because of the condition of compact support in (5.2) the image is larger than just functions of type (5.2).) We will do this by explicitly reducing the integral equation $R_{\mu}^{*} g=h$ to a one-dimensional equation of Volterra type.

Pick $h$ of type (5.2). We need to solve $R_{\mu}^{*} g=h$ for $g$ of type (5.1). That is, we must solve

$$
h\left(k_{0} \cdot H\right)=Y_{\delta}\left(k_{0} \cdot H_{0}\right) h_{\delta}(H)=R_{\mu}^{*} g\left(k_{0} \cdot H\right) .
$$

Computing, we get

$$
\begin{aligned}
& R_{\mu}^{*} g\left(k_{0} \cdot H\right)=\int_{K / M} g\left(k_{0} \cdot H+k \cdot \mathfrak{q}\right) \mu\left(k_{0} \cdot H, k_{0} \cdot H+k \cdot \mathfrak{q}\right) d k \\
&=\int_{K / M} g\left(k_{0} \cdot H+k_{0} k \cdot \mathfrak{q}\right) \mu\left(k_{0} \cdot H, k_{0} \cdot H+k_{0} k \cdot \mathfrak{q}\right) d k \\
&=\int_{K / M} g\left(k_{0} k\left(k^{-1} \cdot H+\mathfrak{q}\right) \mu\left(k_{0} \cdot H, k_{0} k\left(k^{-1} \cdot H+\mathfrak{q}\right)\right) d k\right. \\
&=\int_{K / M} g\left(k_{0} k\left(P_{\mathfrak{a}}\left(k^{-1} \cdot H\right)+\mathfrak{q}\right) \mu\left(H, k\left(P_{\mathfrak{a}}\left(k^{-1} \cdot H\right)+\mathfrak{q}\right)\right) d k\right. \\
&=\int_{K / M} Y_{\delta}\left(k_{0} k \cdot H_{0}\right) g_{\delta}\left(P_{\mathfrak{a}}\left(k^{-1} \cdot H\right)\right) \mu\left(k^{-1} \cdot H, P_{\mathfrak{a}}\left(k^{-1} \cdot H\right)+\mathfrak{q}\right) d k \\
&=\int_{K / M} \int_{M} Y_{\delta}\left(k_{0} m k \cdot H_{0}\right) g_{\delta}\left(P_{\mathfrak{a}}\left(k^{-1} m^{-1} \cdot H\right)\right) \\
&=\int_{K / M} \int_{M} Y_{\delta}\left(k_{0} m k \cdot H_{0}\right) g_{\delta}\left(P_{\mathfrak{a}}\left(k^{-1} \cdot H, P_{\mathfrak{a}}\left(k^{-1} m^{-1} \cdot H\right)+\mathfrak{q}\right) d m d k\right. \\
& \times \mu^{-1} \cdot H\left(k^{-1} \cdot P_{\mathfrak{a}}\left(k^{-1} \cdot H\right)+\mathfrak{q}\right) d m d k \\
&=\int_{K / M} Y_{\delta}\left(k_{0} \cdot H_{0}\right) \varphi_{\delta}\left(k \cdot H_{0}\right) g_{\delta}\left(P_{\mathfrak{a}}\left(k^{-1} \cdot H\right)\right) \\
& \times \mu\left(k^{-1} \cdot H, P_{\mathfrak{a}}\left(k^{-1} \cdot H\right)+\mathfrak{q}\right) d k .
\end{aligned}
$$

The second and sixth equalities follow from the $K$ invariance of $d k$. The 
fourth and fifth equalities follow from the $K$ invariance of $\mu$. The seventh follows because $m \cdot H=H$. The last equality is Lemma 4.1.

Let $H=r H_{0}$. The last integral above then equals

$$
Y_{\delta}\left(k_{0} \cdot H_{0}\right) \int_{K / M} \varphi_{\delta}\left(k \cdot H_{0}\right) g_{\delta}\left(r P_{\mathfrak{a}}\left(k^{-1} \cdot H_{0}\right)\right) \mu\left(r k^{-1} \cdot H_{0}, r P_{\mathfrak{a}}\left(k^{-1} \cdot H_{0}\right)+\mathfrak{q}\right) d k .
$$

Thus, equation (5.3) reduces to

$$
h_{\delta}\left(r H_{0}\right)=\int_{K / M} \varphi_{\delta}\left(k \cdot H_{0}\right) g_{\delta}\left(r P_{\mathfrak{a}}\left(k^{-1} \cdot H_{0}\right)\right) \mu\left(r k^{-1} \cdot H_{0}, r P_{\mathfrak{a}}\left(k^{-1} \cdot H_{0}\right)+\mathfrak{q}\right) d k \text {. }
$$

Using Lemmas 4.3 and 4.2 this equation becomes the following:

$$
\begin{aligned}
& \begin{array}{l}
m_{2 \alpha}>1: \\
h_{\delta}\left(r H_{0}\right)=c \int_{M} \int_{s=-1}^{1} \int_{b=0}^{\sqrt{1-s^{2}}} \varphi_{\delta}\left(m\left(s H_{0}+a Z_{1}+b z_{2}\right)\right) g_{\delta}\left(r s H_{0}\right) \\
\quad \times \mu\left(m_{1} r\left(s H_{0}-a Z_{1}-b Z_{1}\right), r s H_{0}+\mathfrak{q}\right) a^{m_{n}-2} b^{m_{2 n}-1} d b d s d m,
\end{array} \\
& \begin{array}{r}
m_{2 \alpha}=1: \\
h_{\delta}\left(r H_{0}\right)=c \int_{M} \int_{s=-1}^{1} \int_{b=-\sqrt{1-s^{2}}}^{\sqrt{1-s^{2}}} \varphi_{\delta}\left(m\left(s H_{0}+a Z_{1}+b Z_{2}\right)\right)
\end{array} \\
& \quad \times g_{\delta}\left(r s H_{0}\right) \mu\left(m_{1} r\left(s H_{0}-a Z_{1}-b Z_{2}\right), r s H_{0}+\mathfrak{q}\right) a^{m_{n}-2} d b d s d m, \\
& m_{2 \alpha}=0: \quad \times g_{\delta}\left(r s H_{0}\right) \mu\left(m_{1} r\left(s H_{0}-a Z_{1}\right), r s H_{0}+\mathfrak{q}\right) a^{m_{n}-2} d s d m .
\end{aligned}
$$

Because of the homogeneity requirements on $h_{\delta}$ and $g_{\delta}$ we can assume that $r>0$.

We now manipulate these equations as follows:

$$
\begin{aligned}
& m_{2 \alpha}>1: \\
& \begin{aligned}
h_{\delta}\left(r H_{0}\right)=c \int_{s=-1}^{1} \int_{b=0}^{\sqrt{1-s^{2}}} & \varphi_{\delta}\left(\left(s H_{0}+a Z_{1}+b Z_{2}\right)\right) g_{\delta}\left(r s H_{0}\right) \\
& \times \mu\left(r\left(s H_{0}-a Z_{1}-b Z_{2}\right), r s H_{0}+\mathfrak{q}\right) a^{m_{u}-2} b^{m_{2 n}-1} d b d s \\
=2 c \int_{s=0}^{1} \int_{b=0}^{\sqrt{1-s^{2}}} & \varphi_{\delta}\left(\left(s H_{0}+a Z_{1}+b Z_{2}\right)\right) g_{\delta}\left(r s H_{0}\right) \\
& \times \mu\left(r\left(s H_{0}-a Z_{1}-b Z_{2}\right), r s H_{0}+\mathfrak{q}\right) a^{m_{u n}-2} b^{m_{2 n}-1} d b d s .
\end{aligned}
\end{aligned}
$$

The first equality follows from the $M$ invariance of $\varphi_{\delta}$ and $\mu$ and the fact that $M$ centralizes $\mathfrak{a}$. The second equality follows from the homogeneity of $\varphi_{\delta}$ and $g_{\delta}$, the $M$ invariance of $\varphi_{\delta}$, the transitivity of $M$ on the product of the unit spheres of $\mathfrak{q}_{1}$ and $\mathfrak{q}_{2}$, and the $M^{\prime}$ invariance of $\mu$. 
Similarly, for $m_{2 \alpha}=1$,

$$
\begin{aligned}
h_{\delta}\left(r H_{0}\right)=2 c \int_{s=0}^{1} \int_{b=0}^{\sqrt{1-s^{2}}} & {\left[\varphi_{\delta}\left(\left(s H_{0}+a Z_{1}+b Z_{2}\right)\right)+\varphi_{\delta}\left(\left(s H_{0}+a Z_{1}-b Z_{2}\right)\right)\right] } \\
& \times g_{\delta}\left(r s H_{0}\right) \mu\left(r\left(s H_{0}-a Z_{1}-b Z_{2}\right), r s H_{0}+\mathfrak{q}\right) a^{m_{n}-2} d b d s .
\end{aligned}
$$

Here we used all the invariance used above and the hypothesis $\mu(H+Y, H+\mathfrak{q})=$ $\mu(H-Y, H+\mathfrak{q})$.

Finally, for $m_{2 \alpha}=0$ (assuming $m_{c}>1$ ) we get

$h_{\delta}\left(r H_{0}\right)=2 c \int_{s=0}^{1} \varphi_{\delta}\left(\left(s H_{0}+a Z_{1}\right)\right) g_{\delta}\left(r s H_{0}\right) \mu\left(r\left(s H_{0}-a Z_{1}\right), r s H_{0}+\mathfrak{q}\right) a^{m_{n}-2} d s$.

Now make the change of coordinates $y=r s, \beta=r b$ and let $\alpha^{2}=r^{2} a^{2}=$ $r^{2}-y^{2}-\beta^{2}$. Using the homogeneity of $\varphi$ and the assumption that $g_{\delta}$ is supported outside the ball of radius $R$ we get:

For $m_{2 \alpha}>1$ :

$$
\begin{aligned}
h_{\delta}\left(r H_{0}\right)= & 2 c \int_{y=R}^{r} \int_{\beta=0}^{\sqrt{r^{2}-y^{2}}} \varphi_{\delta}\left(y H_{0}+\alpha Z_{1}+\beta Z_{2}\right) g_{\delta}\left(y H_{0}\right) \\
& \times \mu\left(y H_{0}-\alpha Z_{1}-\beta Z_{2}, y H_{0}+\mathfrak{q}\right) \alpha^{m_{n}-2} \beta^{m_{2 n}-1} r^{-\left(m_{n}+m_{2 n}-1+d_{\delta}\right)} d y d \beta .
\end{aligned}
$$

For $m_{2 \alpha}=1$ :

$$
\begin{aligned}
h_{\delta}\left(r H_{0}\right)=2 c \int_{y=R}^{r} & \int_{\beta=0}^{\sqrt{r^{2}-y^{2}}}\left[\varphi_{\delta}\left(y H_{0}+\alpha Z_{1}+\beta Z_{2}\right)+\varphi_{\delta}\left(y H_{0}+\alpha Z_{1}-\beta Z_{2}\right)\right] \\
& \times g_{\delta}\left(y H_{0}\right) \mu\left(y H_{0}-\alpha Z_{1}-\beta Z_{2}, y h_{0}+\mathfrak{q}\right) \alpha^{m_{n}-2} r^{-\left(m_{n}+d_{\delta}\right)} d y d \beta .
\end{aligned}
$$

For $m_{2 \alpha}=0$ :

$$
\begin{aligned}
h_{\delta}\left(r H_{0}\right)=2 c \int_{y=R}^{r} \varphi_{\delta}\left(y H_{0}+\alpha Z_{1}\right) & g_{\delta}\left(y H_{0}\right) \\
& \times \mu\left(y H_{0}-\alpha Z_{1}, y H_{0}+\mathfrak{q}\right) \alpha^{m_{n .}-2} r^{-\left(m_{\bullet .}-1+d_{\delta}\right)} d y .
\end{aligned}
$$

Now let $u^{2}=r^{2}-y^{2}$ (in particular, for $m_{2 \alpha}=0$ we get $u^{2}=\alpha^{2}$ ). Use the equations to define the function $\Phi(u, y)$ such that the following equation holds (for any value of $m_{2 \times}$ ).

$$
r^{\left(m_{n+1}+m_{2 n}-1+d_{\delta}\right)} h_{\delta}\left(r H_{0}\right)=\int_{R}^{r} \Phi\left(\sqrt{r^{2}-y^{2}}, y\right) g_{\delta}\left(y H_{0}\right) d y .
$$

The following Lemma 5.1 and Theorem 5.2 show that if $h_{\delta}\left(r H_{0}\right)$ is smooth in $r$ and compactly supported in $R \leq r \leq \infty$ then we can solve the above equation for $g_{\delta}$. This completes the proof of the support theorem. 
Lemma 5.1. We can write $\Phi(u, y)=u^{m_{11}+m_{2 n}-2} E\left(u^{2}, y\right)$, where $E$ is smooth and $E(0, r) \neq 0$.

Proof. Case a, $m_{2 \alpha} \neq 0$. By definition

$$
\begin{aligned}
\Phi(u, y)=2 c \int_{0}^{u} F\left(y, \sqrt{u^{2}-\beta^{2}}, \beta\right) \mu_{1}\left(y,-\sqrt{u^{2}-\beta^{2}},-\beta\right) \\
\quad \times\left(u^{2}-\beta^{2}\right)^{\left(m_{n}-2\right) / 2} \beta^{m_{2 n}-1} d \beta,
\end{aligned}
$$

where $F\left(y, \sqrt{u^{2}-\beta^{2}}, \beta\right)$ equals

$$
\begin{aligned}
& \varphi_{\delta}\left(y H_{0}+\left(\sqrt{u^{2}-\beta^{2}}\right) Z_{1}+\beta Z_{2}\right) \quad \text { if } m_{2 \alpha}>1, \text { and } \\
& \varphi_{\delta}\left(y H_{0}+\left(\sqrt{u^{2}-\beta^{2}}\right) Z_{1}+\beta Z_{2}\right)+\varphi_{\delta}\left(y H_{0}+\left(\sqrt{u^{2}-\beta^{2}}\right) Z_{1}-\beta Z_{1}\right) \text { if } m_{2 \alpha}=1 .
\end{aligned}
$$

Also $\mu_{1}\left(y,-\sqrt{u^{2}-\beta^{2}},-\beta\right)=\mu\left(y H_{0}-\left(\sqrt{u^{2}-\beta^{2}}\right) Z_{1}-\beta Z_{2}, y H_{0}+\mathfrak{q}\right)$.

Using the $M$ invariance of $\varphi_{\delta}$ and the facts about the transitivity of $M$ stated between Lemmas 4.1 and 4.2 we see that $F$ is even in its second and third variables. Using the $M$ invariance of $\mu$ (and if $m_{2 \alpha}=1$, hypothesis (3.2)(4)) we see that $\mu_{1}$ is also even in its second and third variables. Thus, making the obvious change in notation we get

$\Phi(u, y)=\int_{0}^{u} \widetilde{F}\left(y, u^{2}-\beta^{2}, \beta^{2}\right) \tilde{\mu}\left(y, u^{2}-\beta^{2}, \beta^{2}\right)\left(u^{2}-\beta^{2}\right)^{\left(m_{n}-2\right) / 2} \beta^{m_{2 n}-1} d \beta$,

Since $\varphi_{\delta}$ is a polynomial, $\tilde{F}$ is smooth. By hypothesis $\tilde{\mu}$ is smooth. Thus $\Phi(u, y)$ is also smooth. Also note, since $m_{2 \alpha} \neq 0$, that $m_{\alpha}$ is even.

Using equation $(5.4)$ we differentiate $\Phi(u, y)$, with respect to $u,\left(m_{\alpha}+\right.$ $\left.m_{2 \alpha}-2\right)$ times. We get

$$
\left(\frac{\partial}{\partial u}\right)^{\left(m_{\iota}+m_{2 u}-2\right)} \Phi(0, y)=c \widetilde{F}(y, 0,0) \cdot \tilde{\mu}(y, 0,0)=c \varphi_{\delta}\left(y H_{0}\right) \cdot \mu\left(y H_{0}, y H_{0}+q\right)
$$

for some nonzero constant $c$. (To compute $c$ : note that

$$
c=\left(\frac{\partial}{\partial u}\right)^{\left(m_{n}+m_{2 n}-2\right)} \int_{0}^{u}\left(u^{2}-\beta^{2}\right)^{\left(m_{n}-2\right) / 2} \beta^{m_{2 n}-1} d \beta .
$$

The integral is easily computed to be $c_{1} u^{\left(m_{n}+m_{2, k}-2\right)} \quad\left(m_{\alpha}\right.$ is even $)$. The constant $c_{1}$ is not zero since the integrand is positive. Thus $c=c_{1}\left(m_{\alpha}+m_{2 \alpha}-2\right) ! \neq 0$.) Because of the assumption (3.2)(2) equation (5.5) is never equal to zero. On the other hand all lower-order derivatives with respect to $u$ at $u=0$ are easily seen to be 0 . This proves Case a.

Case $b, m_{2 \alpha}=0$, is immediate from the assumption (3.2).

Theorem 5.2. Let $K(r, y)$ be a smooth function such that $K(r, r) \neq 0$ for all $r \geq 0$. Let $k$ be a nonnegative integer and let $\psi$ be a smooth function with 
$\operatorname{supp} \psi \subseteq[R, \infty]$. Then the equation $\psi(r)=\int_{R}^{r}(r-y)^{k / 2} K(r, y) g(y) d y$ can always be solved for a unique $g(y)$, which is smooth and with supp $g \subseteq[R, \infty]$. Proof. If $k$ is even this is a Volterra equation of the first kind. If $k$ is odd this is Abel's equation. See Yosida [12].

\section{CHANGE OF FIELDS}

So far we have used real planes in $\mathfrak{p}$. In this section we will investigate changing fields. That is, if $\mathfrak{p}$ has a complex or quaternionic structure then we can take the space of complex or quaternionic hyperplanes to be our horocycle space.

Remark. In $\mathbb{H}^{n}$ we will have scalars act on the right. That is, $a \cdot v=v \bar{a}$, for $a \in \mathbb{H}$ and $v \in \mathbb{H}^{n}$.

We consider two series of symmetric spaces, $G / K$ :

(1) $G=\mathrm{SU}(n, 1), K=S(U(n) \times U(1))$.

(2) $G=\operatorname{Sp}(n, 1), K=\operatorname{Sp}(n) \times \operatorname{Sp}(1)$.

We write $\mathbb{F}=\mathbf{C}$ in case (1) and $\mathbb{F}=\mathbb{H}$ in case (2).

Remark. There are other possibilities. For example, we have a complex structure on $\mathbb{H}^{n}$. Also, we have an octonionic structure on $F_{4} / \mathrm{SO}(9)$.

We can make the following identifications:

$$
\begin{aligned}
& \mathbb{X}_{0}=\mathfrak{p}=\mathbb{F}^{n}, \quad H_{0}=\left(\begin{array}{c}
0 \\
\cdot \\
\cdot \\
0 \\
1
\end{array}\right) \in \mathfrak{p}, \quad \mathfrak{a}=\mathbb{R} \cdot H_{0}, \\
& \mathfrak{a}+\mathfrak{q}=\mathbb{F} \cdot H_{0}, \quad \mathfrak{q}_{1}=\left\{\left(\begin{array}{c}
f_{1} \\
\cdot \\
\cdot \\
f_{n-1} \\
0
\end{array}\right) \mid f_{i} \in \mathbb{F}\right\} \quad \text { (an } \mathbb{F} \text { hyperplane). }
\end{aligned}
$$

We consider $\operatorname{Sp}(n)$ to be the group of $n \times n$ matrices with entries in $\mathbb{H}$ preserving the inner product on $\mathbb{H}^{n}$ given by

$$
\langle v, w\rangle=\sum \bar{w}_{i} v_{i}
$$

Take $k=\left(k_{1}, u\right) \in K$ (recall, $K$ is a subgroup of a product group) and $X \in \mathfrak{p}$; then, using the identifications above, we have $k \cdot X=k_{1} X \bar{u} .\left(k_{1} X\right.$ is matrix multiplication)

We let $\Xi_{\mathbb{F}}$ denote the space of affine $\mathbb{F}$ hyperplanes. It is clear that $G_{0}$ acts transitively on $\Xi_{\mathbb{F}}$. This makes $\Xi_{\mathbb{F}}$ a homogeneous space. Define the map

$$
\Phi: \Xi_{0} \rightarrow \Xi_{\mathbb{F}}
$$

by

$$
\Phi(k \cdot(H+\mathfrak{q}))=k \cdot\left(H+\mathfrak{q}_{1}\right) .
$$


Lemma 6.1. The map $\Phi$ is well defined.

Proof. If $k \cdot(H+q)=k_{1} \cdot\left(H_{1}+q\right)$ then $\mathrm{km}=k_{1}$ and $m \cdot H_{1}=H$ for some $m \in M^{\prime}$. Since $m \cdot \mathfrak{q}_{1}=\mathfrak{q}_{1}$ we get $k \cdot\left(H+\mathfrak{q}_{1}\right)=k_{1} \cdot\left(H_{1}+\mathfrak{q}_{1}\right)$.

Lemma 6.2. The map

$\Phi: \Xi_{0} /\{$ hyperplanes through 0$\} \rightarrow \Xi_{\mathbb{F}} /\{\mathbb{F}$ hyperplanes through 0$\}$ is a diffeomorphism.

Proof. Suppose $H \neq 0$ and $H_{1} \neq 0$. (So, $\left.0 \notin k \cdot H+\mathfrak{q}\right)$ etc.) If $k \cdot\left(H+\mathfrak{q}_{1}\right)=$ $k_{1} \cdot\left(H_{1}+\mathfrak{q}_{1}\right)$ then $k_{1}^{-1} k \cdot\left(H+\mathfrak{q}_{1}\right)=H_{1}+\mathfrak{q}_{1}$. Thus, $k_{1}^{-1} k \cdot \mathfrak{q}_{1}=\mathfrak{q}_{1}$ and $k_{1}^{-1} k \cdot H=H_{1}+Y$ for some $Y \in \mathfrak{q}_{1}$. This implies $\left|H_{1}\right| \leq|H|$. By symmetry, $|H|=\left|H_{1}\right|$. This implies $k_{1}^{-1} k \cdot H=H_{1}$. Since $H \neq 0$ we have $k_{1}^{-1} k \in M^{\prime}$. Thus, $k_{1}^{-1} k \cdot \mathfrak{q}=\mathfrak{q}$ and, hence, $k \cdot(H+\mathfrak{q})=k_{1} \cdot\left(H_{1}+\mathfrak{q}\right)$. This shows $\Phi$ is injective.

Let $\widetilde{M}$ be the stabilizer of $\mathfrak{q}_{1}$ in $K$. To show $\Phi$ is surjective we simply have to note that $\widetilde{M}$ acts transitively on the unit sphere in $\mathfrak{a}+\mathfrak{q}_{2}$.

To show $\Phi$ is regular we use the following coordinates. For $0 \neq H \in \mathfrak{a}$ take a small neighborhood $U \subset \mathfrak{a}$. Then $K / M \times U((k, H) \rightarrow k \cdot(H+\mathfrak{q})$ etc. $)$ provides local coordinates for both spaces. With respect to these coordinates $\Phi$ is the identity map. Thus, $\Phi$ is regular.

This lemma shows that for purposes of integration we can use the "coordinates" $K / M \times \mathfrak{a}$ on $\Xi_{\mathbb{F}}$. The measure we will use is $c|H|^{m_{2 n}} d k d H$. (It is $G_{0}$ invariant.) Here, $c$ is the area of the unit sphere in $\mathfrak{a}+\mathfrak{q}_{2}$.

The next proposition is best developed using group theoretic notation. As before, let $\widetilde{M}$ be the stabilizer of $\mathrm{q}_{1}$ in $K$. Similar to the real case, we have

$$
G_{0} / \widetilde{M} \approx\{(X, \xi) \mid X \in \xi\} \quad\left(g \widetilde{M} \leftrightarrow\left(g \cdot 0, g \cdot \mathfrak{q}_{1}\right)\right) .
$$

This gives the double fibration

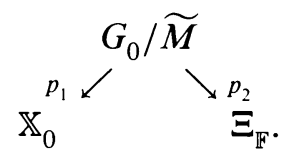

As in $\S 1$, we get $\check{X}=p_{2} p_{1}^{-1}(X)$ and $\hat{\xi}=p_{1} p_{2}^{-1}(\xi)$.

For $\mu \in C\left(G_{0} / \widetilde{M}\right)$ we define

$$
{ }_{\mathrm{F}} R_{\mu} f(\xi)=\int_{\mathfrak{q}_{1}} f\left(g \cdot T_{Y} \cdot 0\right) \mu\left(g \cdot T_{Y} \widetilde{M}\right) d Y
$$

and

$$
{ }_{\mathbb{F}} R_{\mu}^{*} \varphi(X)=\int_{K} \varphi\left(T_{X} k \cdot \mathfrak{q}_{1}\right) \mu\left(T_{X} k \widetilde{M}\right) d k .
$$

The next lemma describes the duality between ${ }_{\mathbb{F}} R_{\mu}$ and ${ }_{\mathbb{F}}^{*}$. 
Lemma 6.3. Let $d X$ be the usual measure on $\mathfrak{p}$ and, as before, let

$$
c|H|^{m_{2 n}} d H d k=d \xi
$$

be the measure on $\Xi_{\mathbb{F}}$. Then

$$
\int_{\Xi_{\bar{F}}} R_{\mu} f(\xi) \varphi(\xi) d \xi=\int_{\mathfrak{p}} f(X)_{\mathbb{F}} R_{\mu}^{*} \varphi(X) d X .
$$

Proof. The right-hand side is

$$
\begin{aligned}
& \int_{\mathfrak{p}} \int_{K} f(X) \varphi\left(X+k \cdot \mathfrak{q}_{1}\right) \mu\left(T_{X} k \widetilde{M}\right) d k d X \\
& =\int_{K} \int_{\mathfrak{p}} f(k \cdot X) \varphi\left(k \cdot\left(X+\mathfrak{q}_{1}\right) \mu\left(T_{k \cdot X} k \widetilde{M}\right) d X d k\right. \\
& =\int_{K} \int_{\mathfrak{a}+\mathfrak{q}_{2}} \int_{\mathfrak{q}_{1}} f\left(k \cdot\left(H+Y_{2}+Y_{1}\right)\right) \varphi\left(k \cdot\left(H+Y_{2}+\mathfrak{q}_{1}\right)\right) \\
& \times \mu\left(T_{H+Y_{2}+Y_{1}} \widetilde{M}\right) d H d Y_{2} d Y_{1} d k \\
& =c \int_{K} \int_{\mathfrak{a}} \int_{\widetilde{M}} \int_{\mathfrak{q}_{1}} f\left(k \cdot\left(\tilde{m} \cdot H+Y_{1}\right)\right) \varphi\left(k \cdot\left(\tilde{m} \cdot H+\mathfrak{q}_{1}\right)\right) \\
& \times \mu\left(T_{\dot{m} \cdot H+Y_{!}} \widetilde{M}\right)|H|^{m_{2 n}} d \tilde{m} d H d Y_{1} d k \\
& =c \int_{K} \int_{\mathfrak{a}} \int_{\widetilde{M}} \int_{\mathfrak{q}_{1}} f\left(k \cdot \tilde{m} \cdot\left(H+Y_{1}\right)\right) \varphi\left(k \cdot \widetilde{M} \cdot\left(H+\mathfrak{q}_{1}\right)\right) \\
& \times \mu\left(T_{H+Y_{1}} \widetilde{M}\right)|H|^{m_{2 n}} d \tilde{m} d H d Y_{1} d k \\
& =c \int_{K} \int_{\mathfrak{a}} \int_{\mathfrak{q}_{1}} f\left(k \cdot\left(H+Y_{1}\right)\right) \varphi\left(k \cdot\left(H+\mathfrak{q}_{1}\right)\right) \mu\left(T_{H+Y_{1}} \widetilde{M}\right)|H|^{m_{2 n}} d H d Y_{1} d k \\
& =\int_{\Xi_{\xi}} R_{\mu} f(\xi) \varphi(\xi) d \xi \text {. }
\end{aligned}
$$

Since $\widetilde{M} \supset M^{\prime}$ we have

$$
\mu \in C\left(G_{0} / \widetilde{M}\right) \subset C\left(G_{0} / M^{\prime}\right) .
$$

(We consider $\mu$ as a function on $G_{0}$ invariant by $\widetilde{M}$.) Thus, we also have $R_{\mu}^{*}$ acting on functions on $\Xi_{0}$. The next lemma makes the connection between transforms. From now on we will consider $\mu$ as a function on $G_{0}$ with certain invariance properties.

Lemma 6.4. For any $\varphi \in C\left(\Xi_{0}\right)$ there is a $\tilde{\varphi} \in C\left(\Xi_{\mathbb{F}}\right)$ such that $R_{\mu}^{*} \varphi={ }_{\mathbb{F}} R_{\mu}^{*} \tilde{\varphi}$. If $\varphi$ is smooth then so is $\tilde{\varphi}$.

Proof. Let $\tilde{\varphi}\left(g \cdot \mathfrak{q}_{1}\right)=\int_{\widetilde{M}} \varphi(g \tilde{m} \cdot \mathfrak{q}) d \tilde{m}$. Clearly $\tilde{\varphi}$ is defined on $G_{0} /\left(\widetilde{M} \ltimes \mathfrak{q}_{1}\right)=$ 
$\Xi_{\mathbb{F}}$. Also,

$$
\begin{aligned}
{ }_{\mathbb{F}} R_{\mu}^{*} \tilde{\varphi}(X) & =\int_{K} \tilde{\varphi}\left(T_{X} k \cdot \mathfrak{q}_{1}\right) \mu\left(T_{X} k\right) d k \\
& =\int_{K} \int_{\widetilde{M}} \varphi\left(T_{X} k \tilde{m} \cdot \mathfrak{q}\right) \mu\left(T_{X} k\right) d \tilde{m} d k \\
& =\int_{\widetilde{M}} \int_{K} \varphi\left(T_{X} k \cdot \mathfrak{q}\right) \mu\left(T_{X} k \tilde{m}^{-1}\right) d \tilde{m} d k \\
& =\int_{K} \varphi\left(T_{X} k \cdot \mathfrak{q}\right) \mu\left(T_{X} k\right) d k \\
& =R_{\mu}^{*} \varphi(X) .
\end{aligned}
$$

The third equality follows from the $\widetilde{M}$ invariance $d k$ and the fourth is from the $\widetilde{M}$ invariance of $\mu$.

The second statement in the lemma is clear.

Remark. Clearly, if $\operatorname{supp} \varphi \cap B^{R}=\varnothing$ then $\operatorname{supp} \tilde{\varphi} \cap B^{R}=\varnothing$. Here, the balls are in $\Xi_{0}$ and $\Xi_{\mathrm{F}}$ respectively. (Recall, the norm of a plane in either space is defined to be the distance of the plane to the origin.)

The following corollary is the main result in this section, it generalizes Quinto [10].

\section{Corollary 6.5. Suppose $\mu$ satisfies}

(1) $\mu$ is smooth.

(2) $\mu$ is $K$ invariant $\left(\mu \in C^{\infty}\left(K \backslash G_{0} / \widetilde{M}\right)\right)$.

(3) $\mu\left(T_{H}\right) \neq 0$ for all $H \in \mathfrak{a}$.

Then (1) if $f \in L_{c}^{2}\left(\mathbb{X}_{0}\right)$ and supp ${ }_{\mathbb{F}} R_{\mu} f \subset B^{R}$ then $\operatorname{supp} f \subset B^{R}$,

(2) ${ }_{\mathbb{F}} R_{\mu}$ is injective on $L_{c}^{2}\left(\mathbb{X}_{0}\right)$.

Proof. Part (1): The proof proceeds just like that of Theorem 3.2. By Lemma 6.4 and the proof of Theorem 3.2 every function of type $(5.2)(h(k \cdot H)=$ $\left.Y_{\delta}\left(k \cdot H_{0}\right) h_{\delta}(H) \cdots\right)$ is ${ }_{\mathbb{F}} R_{\mu}^{*} \tilde{\varphi}$ for some $\varphi$ of type (5.1).

Suppose $\operatorname{supp}_{\mathbb{F}} R_{\mu} \tilde{\varphi} \subset B^{R}, h$ is of type (5.2), and $\varphi$ is chosen as above. Then,

$$
\langle f, h\rangle=\left\langle f,{ }_{\mathbb{F}} R_{\mu}^{*} \tilde{\varphi}\right\rangle=\left\langle{ }_{\mathbb{F}} R_{\mu} f, \tilde{\varphi}\right\rangle=0 .
$$

The third bracket is the $L^{2}$ inner product with respect to the measure on $\Xi_{\mathbb{F}}$ from Lemma 6.3. It equals zero by the remark following Lemma 6.4.

As in Theorem 3.2, part (2) follows from part (1) by letting $R$ go to zero.

Remarks. (1) As before, the condition $\mu\left(T_{H}\right) \neq 0$ combined with $K$ invariance says that $\mu(X, \xi) \neq 0$ if $X$ is the point of $\xi$ nearest the origin.

(2) It is interesting to note that Quinto [10] reduces the proof of the support theorem to a Volterra equation and we reduce it to Abel's equation. The difference comes from the way the $\widetilde{M}$ invariance is applied. 


\section{THE SUPPORT THEOREM FOR DISTRIBUTIONS}

In this section we will extend the support theorem to distributions. Most likely, Quinto's argument via elliptic operators (Quinto [11]) would carry through here. However, we will use a different approach via the representation theory of $K$ on $\mathfrak{p}=\mathbb{X}_{0}$.

For $T \in \mathscr{E}^{\prime}(\mathfrak{p})$ we define $R_{\mu} T(\varphi)=T\left(R_{\mu}^{*} \varphi\right)$. This is well defined since $R_{\mu}^{*}$ is continuous on $C^{\infty}\left(\Xi_{0}\right)$.

Part (1) of the following theorem appears in [3, Theorem 2.20] in the case $\mu \equiv 1$. The entire theorem is a generalization of a result in [10].

Theorem 7.1. Suppose $\mu$ satisfies the hypothesis in Theorem 3.2 (see equation (3.2)). Then

(1) If $T \in \mathscr{E}^{\prime}\left(\mathbb{X}_{0}\right)$ and $\operatorname{supp} R_{\mu} T \subset B^{R}$ then $\operatorname{supp} T \subset B^{R}$.

(2) The operator $R_{\mu}: \mathscr{E}^{\prime}(\mathfrak{p}) \rightarrow \mathscr{E}^{\prime}\left(\Xi_{0}\right)$ is injective.

Proof. Part (1): The proof of Theorem 3.2(1) shows $R_{\mu}^{*}$ maps functions in $C^{\infty}\left(\Xi_{0}\right)$ supported outside the ball of radius $R$ onto a dense subspace of functions in $C^{\infty}\left(\mathbb{X}_{0}\right)$ supported outside the ball of radius $R$. This proves part (1).

For part (2), by letting $R \rightarrow 0$, it is enough to show that

$$
R_{\mu}: \text { Distributions supported at } 0 \rightarrow \mathscr{E}^{\prime}\left(\Xi_{0}\right)
$$

is injective. Let $T f=D f(0)$ be an arbitrary distribution supported at the origin. ( $D$ is a constant coefficient differential operator on p.) Suppose $R_{\mu} T=$ 0 ; then $\left.D R_{\mu}^{*} \varphi\right|_{X=0}=0$ for every $\varphi \in C^{\infty}\left(\Xi_{0}\right)$. Lemma 7.3 shows this is impossible. This proves Theorem 7.1.

Before proving Lemma 7.3 we must review some notation and facts concerning polynomials and differential operators on $\mathfrak{p}$. Let $S\left(\mathfrak{p}^{*}\right)$ denote the polynomial algebra of $\mathfrak{p}$. Let $Q(X)=\langle X, X\rangle$. Let $J$ be the subalgebra generated by $Q . J$ is the algebra of $K$-invariant polynomials. As before, let $\mathscr{H}$ be the space of harmonic polynomials. An important result of Rallis (see [9]) says $S\left(\mathfrak{p}^{*}\right)=\mathscr{H} \otimes J$. As in $\S 4, \mathscr{H}=\bigoplus \mathscr{H}_{\delta}$ and we consider elements in $\mathscr{H}$ as either polynomials on $\mathfrak{p}$ or functions on $K / M$. Let $S(\mathfrak{p})$ be the symmetric algebra of $\mathfrak{p}$. We will also think of $S(\mathfrak{p})$ as the algebra of constant coefficient differential operators on $\mathfrak{p}$. Of course, $K$ acts on $S(\mathfrak{p})$ and the Killing form induces a $K$-equivariant isomorphism, $\Psi$, between $S\left(\mathfrak{p}^{*}\right)$ and $S(\mathfrak{p})$. For each $\delta \in \widehat{K}_{M}$ let $V_{\delta}$ be the representation space with unitary structure $\langle,\rangle_{\delta}$. Fix a $K$ equivariant isomorphism $j_{\delta}: V_{\delta} \rightarrow \mathscr{H}_{\delta}$. We will drop the subscript $\delta$ in places where there is no danger of confusion. For each $\delta$ fix a unit vector $v_{\delta} \in V_{\delta}$ which is $M$ invariant and such that $j\left(v_{\delta}\right)=\varphi_{\delta}$. Thus, $j_{\delta}(v)(k)=\left\langle v, k \cdot v_{\delta}\right\rangle$. Finally, let $D_{\delta}=\Psi\left(\varphi_{\delta}\right)$ and $L=\Psi(Q)=$ the Laplacian. 
To summarize,

$$
\begin{array}{lll}
Q(X)=\langle X, X\rangle, & J=\mathbb{C}[Q], & L=\Psi(Q), \\
\Psi: S\left(\mathfrak{p}^{*}\right) \rightarrow S(\mathfrak{p}) ; & S\left(\mathfrak{p}^{*}\right)=\mathscr{H} \otimes J, & \mathscr{H}=\bigoplus \mathscr{H}_{\delta},
\end{array}
$$$$
j_{\delta}: V_{\delta} \rightarrow \mathscr{H}_{\delta}, \quad v_{\delta}=M \text { invariant unit vector, }
$$$$
j_{\delta}\left(v_{\delta}\right)=\varphi_{\delta}, \quad \Psi\left(\varphi_{\delta}\right)=D_{\delta} .
$$

Lemma 7.2. (1) Suppose $P \in \mathscr{H}_{\delta}$ and $D=\Psi(P)$. Then (bar indicates complex conjugation)

$$
\int_{K} \overline{P(K)}\left(k^{-1} \cdot D\right) d k=c D_{\delta}
$$

for some $c \neq 0$.

(2) Suppose $P \in \mathscr{H}_{\delta}, P^{\prime} \in \mathscr{H}_{\delta^{\prime}}, D=\Psi(P)$, and $\delta \neq \delta^{\prime}$. Then

$$
\int_{K} \overline{P^{\prime}(k)}\left(k^{-1} \cdot D\right) d k=0 .
$$

Proof. (1) Choose $v \in V_{\delta}$ such that $j(v)=P$. (So, $P(k)=\left\langle v, k \cdot v_{\delta}\right\rangle$.) Thus,

$$
\begin{aligned}
\int_{K} \overline{P(k)}\left(k^{-1} \cdot D\right) d k & =\Psi \circ j\left(\int_{K} \overline{\left\langle v, k \cdot v_{\delta}\right\rangle} k^{-i} \cdot v d k\right) \\
& =\Psi \circ j\left(\int_{K} \overline{\left\langle k \cdot v, v_{\delta}\right\rangle} k \cdot v d k\right) \\
& =\Psi \circ j\left(c_{1}\langle v, v\rangle v_{\delta}\right) \quad\left(c_{1}=(\operatorname{dim} \delta)^{-1}\right) \\
& =c_{1}\langle v, v\rangle D_{\delta} .
\end{aligned}
$$

The third equality is just the Schur orthogonality relations.

Part (2) is also just the orthogonality relations.

Recall, we can consider $S(\mathfrak{p})$ to be the algebra of constant coefficient differential operators.

Lemma 7.3. Suppose $D=\sum_{\delta, j} D_{\delta, j} L^{j}$, where each $D_{\delta, j} \in \mathscr{H}_{\delta}$. Then there is a $\varphi \in C^{\infty}\left(\Xi_{0}\right)$ such that $D R_{\mu}^{*} \varphi(0) \neq 0$.

Proof. Choose a $\delta$ such that $D_{\delta, j} \neq 0$ for some $j$. Let $N$ be the largest $j$ for which this is true. Take $P \in \mathscr{H}_{\delta}$ such that $\Psi(P)=D_{\delta, N}$. Define $\varphi$ by

$$
\varphi\left(k \cdot\left(r H_{0}+\mathfrak{q}\right)\right)=\overline{P(k)} g\left(r H_{0}\right)=\overline{P(k)} r^{d_{\delta}+2 N} .
$$

We get

$$
\begin{aligned}
D R_{\mu}^{*} \varphi(0) & =D\left(\int_{K} \varphi(X+k \cdot \mathfrak{q}) \mu\left(T_{X} k\right) d k\right)_{X=0} \\
& =D\left(\int_{K} \varphi\left(k \cdot\left(P_{\mathfrak{a}}\left(k^{-1} \cdot X\right)+\mathfrak{q}\right) \mu\left(k T_{k^{-1} \cdot X}\right) d k\right)_{X=0}\right. \\
& =\int_{K} \overline{P(k)}\left(k^{-1} \cdot D\right)\left(g\left(P_{\mathfrak{a}}(X) \mu\left(T_{X}\right)\right)_{X=0} d k\right. \\
& =\left(c_{N} D_{\delta} L^{N}+c_{N-1} D_{\delta} L^{N-1}+\cdots+c_{0} D_{\delta}\right)\left(g\left(P_{\mathfrak{a}}(X)\right) \mu\left(T_{X}\right)\right)_{X=0}
\end{aligned}
$$


The third equality follows from the $K$ invariance of $\mu$ and the definition of the action of $K$ on differential operators. The last equality follows from Lemma 7.2, which guarantees $c_{N} \neq 0$.

The polynomial (in $X$ ) $g \circ P_{\mathfrak{a}}(X)$ is homogeneous of degree $d_{\delta}+2 N$. Since $D_{\delta}$ is homogeneous of degree $d_{\delta}$ and $L$ is homogeneous of degree 2 the last equation above implies $D R_{\mu}^{*} \varphi(0)=c_{N} \mu\left(T_{0}\right)\left(D_{\delta} L^{N} g \circ P_{\mathfrak{a}}\right)(0)$.

We know $\varphi_{\delta}\left(H_{0}\right)=1$ and $\varphi_{\delta}$ is homogeneous of degree $d_{\delta}$. Thus, for $Y \in \mathfrak{q}$ we have $\varphi_{\delta}\left(r H_{0}+Y\right)=r^{d}+R\left(r H_{0}+Y\right)$ where $R(Y)=0$. This shows (as an element of $S(\mathfrak{p})) \quad D_{\delta} \in H_{0}^{d}+S(\mathfrak{p}) \mathfrak{q}$. Similarly, $L \in H_{0}^{2}+S(\mathfrak{p}) \mathfrak{q}$. Thus, $D_{\delta} L^{N} g \circ P_{\mathfrak{a}}\left(r H_{0}+T\right)=\left(d_{\delta}+2 N\right) !$. This proves the lemma.

Corollary 7.4. If $\mu$ satisfies the hypothesis of Corollary 6.5 then

(1) If $T \in \mathscr{E}^{\prime}\left(\Xi_{\mathbb{F}}\right)$ and $\operatorname{supp} R_{\mu} T \subset B^{R}$ then $\operatorname{supp} T \subset B^{R}$.

(2) ${ }_{\mathbb{F}} R_{\mu}: \mathscr{E}^{\prime}\left(X_{0}\right) \rightarrow \mathscr{E}^{\prime}\left(\Xi_{\mathbb{F}}\right)$ is injective.

Proof. Part (1) follows from Lemma 6.4 and the proof of Theorem 7.1.

As in the proof of Theorem 7.1, part (2) reduces to showing ${ }_{\mathbb{F}} R_{\mu}$ is injective on distributions supported at the origin. This follows from the proof of Theorem 7.1 using Lemma 6.4.

\section{REFERENCES}

1. I. M. Gelfand, M. I. Graev, and Z. Ya. Shapiro, Differential forms and integral geometry, Functional Anal. Appl. 3 (1969), 101-114.

2. V. Guillemin, The Radon transform on Zoll surfaces, Adv. in Math. 22 (1976), 85-119.

3. S. Helgason, Groups and geometric analysis, Academic Press, New York, 1984.

4. $\ldots$, Differential geometry, Lie groups and symmetric spaces, Academic Press, New York, 1978.

5. _ A duality for symmetric spaces with applications to group representations, Adv. in Math. 5 (1970), 1-154.

6. __ The Radon transform on Euclidean spaces, compact two point homogeneous spaces, and Grassman manifolds, Acta Math. 113 (1965), 153-180.

7. S. Helgason, A duality in integral geometry on symmetric spaces, Proc. U. S.-Japan Seminar in Differential Geometry (Kyoto, 1965), Nippon Hyronsha, Tokyo, 1966.

8. B. Kostant, On the existence and irreducibility of certain series of representation, Lie Groups and Their Representations (I. M. Gelfand, ed.), Halsted, New York, 1975, pp. 231-329.

9. B. Kostant and S. Rallis, Orbits and Lie group representations on symmetric spaces, Amer. J. Math. 93 (1971), 753-809.

10. E. Quinto, The injectivity of rotation, invariant Radon transforms on complex hyperplanes in $\mathbb{C}^{n}$, preprint, 1987.

11. _ The invertibility of rotation invariant Radon transforms, J. Math. Anal. Appl. 91 (1983), 510-521.

12. K. Yosida, Lectures on differential and integral equations, Interscience, New York, 1960.

Mathematical Sciences Research Institute, Berkeley, California 94720

Current address: Department of Mathematics, Tufts University, Medford, Massachusetts 02155 